\title{
GA-tuning of Multivariable PID Controller for Electrohydraulic Load-Sensing Servo System
}

\author{
Alexander Mitov ${ }^{1, *}$, Tsonyo Slavov ${ }^{2}$, Jordan Kralev ${ }^{2}$ and Ilcho Angelov ${ }^{1}$ \\ ${ }^{1}$ Technical University of Sofia, Dept. of Hydroaerodynamics and Hydraulic Machines, Kliment \\ Ohridski blvd. №8, Bulgaria \\ ${ }^{2}$ Technical University of Sofia, Dept. of Systems and Control, Kliment Ohridski blvd. №8, Bulgaria
}

\begin{abstract}
The main purpose of this article is to present the design, optimal setting and experimental study of a multivariable PID controller supposed for electrohydraulic power steering system with load-sensing function. The set of the controller is performed through an optimization procedure based on a genetic algorithm. The PID controller is embedded into a microcontroller for mobile machinery used in laboratory test rig for investigation of an electrohydraulic servo steering system. Results obtained in conditions of physical experiment and numerical simulation with the optimally tuned PID controller are presented. An analysis of the control performance, as well as some features of the used method for setting the PID controller.
\end{abstract}

\section{Introduction}

Recently, there has been a development of hydraulic drives, which can be summarized in two main directions: the first direction is to introduce digital control, and the second to improve their load-sensitive function. The main architectures of hydraulic systems with load-sensing function are relatively well studied, as well as their selection depending on the desired functionality of the driven machine. In a number of applications of the hydraulic drive, however, the two directions of development are combined, and the ultimate goal is to increase the energy efficiency of the entire electrohydraulic system. Achieving this ultimate goal is impossible without an effective control system [1]. The introduction of digital control in almost all applications of hydraulic drives necessitated the use of embedded control systems. In this aspect, the behavior of the whole system strongly depends on the embedded controller. When there are limitations to the architecture of the hydraulic system (i.e. it is solved), then efforts are focused on achieving high levels of control performance. It could be tentatively assumed that such a case is observed in electrohydraulic servo systems for power steering of mobile machines.

The task of controlling electrohydraulic servo systems in most of their variants can be solved by a classical PID control law obtained from an adequate model and set by an appropriate algorithm [2]. Evidenced by a number of studies and developments by other authors. Rather, efforts are focused on developing the methods and tuning algorithms used

\footnotetext{
* Corresponding author: a mitov@tu-sofia.bg
} 
in them. One of the modern algorithms used for optimal setting of PID controllers is the socalled Genetic algorithm (GA). In [3] an improved genetic algorithm (IGA) to search for the optimal PID gains for robotic excavator trajectory control is proposed.

The authors have accumulated experience in the design and implementation of robust embedded controllers with application not only in electrohydraulic drive systems. Their previous developments have studied various types, both conventional and multivariable controllers of electrohydraulic systems for power steering systems without load-sensing. The present study focuses on a load-sensing power steering system. For this purpose, a state space model of the system is derived on the basis of an identification procedure based on experimental data shown in detail in [4]. The PID controller implements the state-space model of the electro-hydraulic servo actuator system to control the movable surface of space vehicle are presented in [5] and genetic algorithm (GA) is used to optimize the controller gain parameters. In [6] proposes the tuning method by GA which approached the optimal PID parameters so that it can increase the efficiency control of the electro-hydraulic servo valve system. A similar approach has been used in [7]. However, all these mentioned developments use the Ziegler-Nichols method to determine the initial values for of the PID controller gains, which is again a classical approach for single input single output system (SISO). In the present work, this approach for determining the initial conditions is inapplicable because the system under study is a multivariable which is represented as single input and multiple output (SIMO).

The main purpose of this article is to present the design, optimal setting and experimental study of a multivariable PID controller designed for electrohydraulic power steering system with load sensitivity. The set of the controller is performed through an optimization procedure based on a GA. The PID controller is embedded into a microcontroller for mobile machinery used in laboratory test rig for investigation of a electrohydraulic servo steering system. Results obtained in conditions of physical experiment and numerical simulation with the optimally tuned PID controller are presented. An analysis of the control performance, as well as some features of the used method for setting the PID controller.

\section{Hardware description}

The authors have designed and realized a new laboratory test rig for investigation of LS servo system applicable for power steering low-speed mobile machines. The hydraulic circuit diagram of the system synthesis on the laboratory model is depicted in Fig.1. The detailed description of the studied system is presented in a previous paper [4].

\section{Identified model}

To obtain a model of electrohydraulic servo system the two analytical modelling and system identification approaches can be used [9]. In our case, due to the lack of information about numerical value of parameters of internal units of electrohydraulic steering system, the identification approach is preferred. The additional advantages of models determined by "black box" identification are that the description of noises, actuators and sensor dynamics are obtained. For that reason, we chose to estimate numerical model of the plant by system identification procedure which is detail presented in [4]. It is assumed that the dynamics of electro-hydraulic steering system may be described in sufficiently wide operating range by linear discrete time stochastic state space model

$$
\begin{aligned}
& x(k+1)=A x(k)+B u(k)+K e(k) \\
& y(k)=C x(k)+D u(k)+e(k),
\end{aligned}
$$


where $x=\left[\begin{array}{llll}x_{1} & x_{2} & \ldots & x_{n}\end{array}\right]^{T}$ is a state vector, $u$ is a control signal that is a voltage applied to the control input of PVE, $y=\left[\begin{array}{ll}y_{L V D T} & y_{p o s}\end{array}\right]^{T}$ is output signals ( $y_{L V D T}$ is output of LVDT sensor and $y_{p o s}$ is a cylinder piston position), $e(k)=\left[\begin{array}{ll}e_{1} & e_{2}\end{array}\right]^{T}$ is a residual vector $\left(e_{1}\right.$ and $e_{2}$ are residuals respect to the LVDT and cylinder positon respectively). $A, B, C, D$ and $K$ are the matrices (model parameters) with appropriate dimensions.

The state space models (1) of various orders are estimated. Model parameters are determined by model predictive error method for estimation of state space model. In estimation, the observable canonical form of state pace model (1) is used. The best results are obtained with model of $4^{\text {th }}$ order. The values of estimated parameters are

and covariance matrix

$$
\begin{aligned}
A & =\left(\begin{array}{cccc}
0 & 1 & 0 & 0 \\
-2.1432 & 2.1207 & 1.4618 & -1.4624 \\
0 & 0 & 0 & 1 \\
-0.3563 & -0.3524 & 0.0464 & 0.9532
\end{array}\right), \\
B & =10^{-5}\left(\begin{array}{c}
0.0231 \\
0.4533 \\
0.2308 \\
-0.4971
\end{array}\right), C=\left(\begin{array}{llll}
1 & 0 & 0 & 0 \\
0 & 0 & 1 & 0
\end{array}\right) \\
D & =\left(\begin{array}{l}
0 \\
0
\end{array}\right), K=\left(\begin{array}{cc}
1.5999 & -0.0031 \\
1.7777 & -0.0084 \\
-0.5023 & 0.9847 \\
-1.4377 & 0.9866
\end{array}\right)
\end{aligned}
$$

$$
M\left(e e^{T}\right)=\left(\begin{array}{cc}
0.0039 & -0.0007 \\
-0.0007 & 0.4739
\end{array}\right)
$$

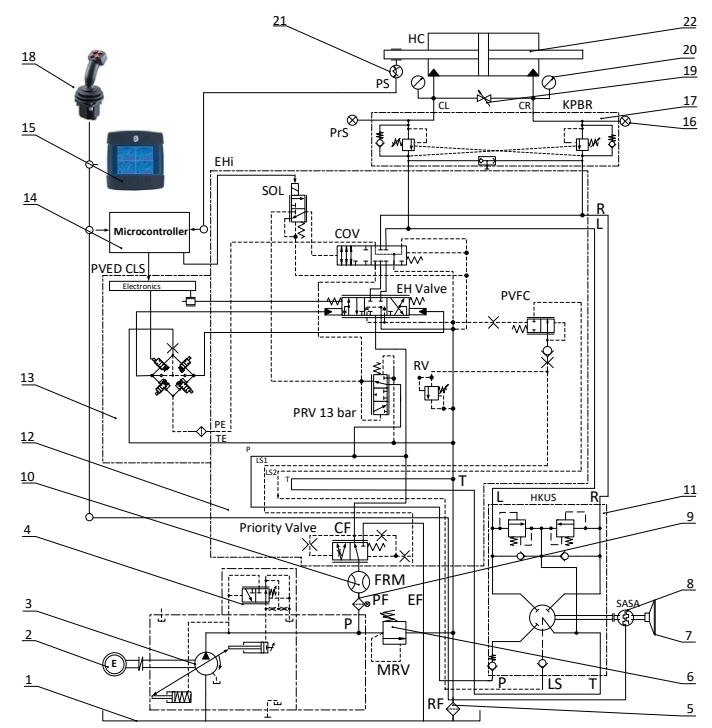

Fig.1. Hydraulic circuit diagram of a new laboratory test rig. 


\section{GA tuning of multivariable PID controller}

The block scheme of control system is presented in Fig.2. The design of PID controller is based on the model (3).

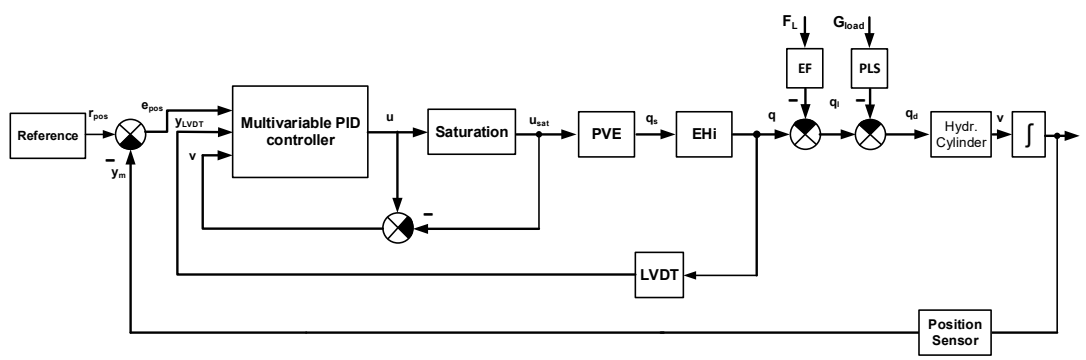

Fig.2. Block Scheme of control system.

The description of popular in practice single input single output parallel PID controller with low pass filter in derivative term and anti-wind up mechanism is given by

$$
u(s)=K_{p}\left(\operatorname{err}(s)+\frac{T_{d} N s}{s+N} \operatorname{err}(s)\right)+\left(K_{p} K_{i} \operatorname{err}(s)-k_{w} v(s)\right) \frac{1}{s}, v(s)=u(s)-u_{s a t}(s),
$$

where $K_{p}$ is the proportional gain, $K_{i}$ is the integral gain, $T_{d}$ is the derivative time constant, $N$ is the pole of the low pass filter, $k_{w}$ is the conditional feedback gain used to realize anti wind up algorithm, $\operatorname{err}(s)=r(s)-y(s)$ is reference error. The $u_{s a t}(s)$ is saturated control signal which is obtained as

$$
u_{\text {sat }}=\left\{\begin{array}{c}
u(s), u_{\min } \leq u \leq u_{\max } \\
u_{\min }, u<u_{\min } \\
u_{\max }, u>u_{\max }
\end{array},\right.
$$

where $u_{\min }$ and $u_{\max }$ are minimal and maximal admissible values of control signal. In order to implement the PID controller in digital control device, the integral and derivative terms in equation (4) are discretized by first order forward difference and first order backward difference respectively. Thus, the discrete-time PID controller is described by

$$
u(z)=K_{p}\left(e(z)+\frac{T_{d} N(z-1)}{\left(1+N T_{s}\right) z-1} e(z)\right)+\left(K_{p} K_{i} e(z)-k_{w} v(z)\right) \frac{T_{s}}{(z-1)},
$$

where $T_{s}$ is a sample time. Based on equation (6) the multivariable PID controller for electrohydraulic load-sensing servo system is designed. It has three inputs and one output. The first input is position error $e_{p o s}=r_{p o s}-y_{p o s}$ and the second one is $y_{L V D T}$, where $r_{p o s}$ is reference for cylinder piston position. The third input is the signal form conditional feedback $v$. The designed discrete time multivariable PID controller is described by 


$$
u(z)=\left[\begin{array}{ccc}
K_{p_{p o s}} & K_{p_{L V D T}} & 0 \\
K_{p_{p o s}} K_{i_{p o s}} \frac{T_{s}}{(z-1)} & 0 & \frac{T_{s}}{(z-1)} \\
\frac{K_{p_{p o s}} T_{d_{p o s}} N_{p o s}(z-1)}{\left(1+N_{p o s} T_{s}\right) z-1} & \frac{K_{p_{L V D T}} T_{d_{L V D T}} N_{L V D T}(z-1)}{\left(1+N_{L V D T} T_{s}\right) z-1} & 0
\end{array}\right]\left[\begin{array}{c}
e_{p o s}(z) \\
-y_{L V D T}(z) \\
v(z)
\end{array}\right]\left[\begin{array}{l}
1 \\
1 \\
1
\end{array}\right],
$$

where $T_{s}=0.01 \mathrm{~s}, u_{\min }=-5000, u_{\max }=5000$ which are used to evaluate signal $v$ according to equation (5). $K_{p_{p o s}}, K_{p_{L V D T}}, K_{i_{p o s}}, T_{d_{p o s}}, T_{d_{L V D T}}$ are controller parameters and poles of low pass filters are chosen as $N_{p o s}=\frac{T_{d_{p o s}}}{5}, N_{L V D T}=\frac{T_{d_{L V D T}}}{5}$. As can be seen from equation (7) for feedback of cylinder piston position the PID control law is used, whereas for feedback of LVDT sensor the PD control law is implemented. The design problem of PID controller (7) for model (3) is to find the vector with unknown parameters $\theta=\left[\begin{array}{lllll}K_{p_{p o s}} & K_{p_{L V D T}} & K_{i_{p O s}} & T_{d_{p O s}} & T_{d_{L V D T}}\end{array}\right]^{T}$, which provide desired control system performance. A useful method to obtain parameters is optimal tuning. The performance index to be minimized, is given by

$$
\min _{\theta} J(\theta), J(\theta)=\sum_{i=0}^{N^{*}} e_{p o s}^{2}(i, \theta)+R \sum_{i=0}^{N^{*}} u^{2}(i, \theta),
$$

where the weighting coefficient $R=0.001$ is experimentally chosen in order to obtain acceptable form of control signal. The unconstrained optimization problem (8) can be solved by various classical and modern methods [10]. In case of objective functions with several local minima's a methods for global optimization such as firefly optimization, simulated annealing, pattern search and genetic algorithm are preferred. The last one is especially suitable in case of unknown initial parameters, which is the case in this paper.

The genetic algorithm is based on the natural selection, which is a method that drives biological evolution. The block scheme of genetic algorithm is depicted in Fig.3.

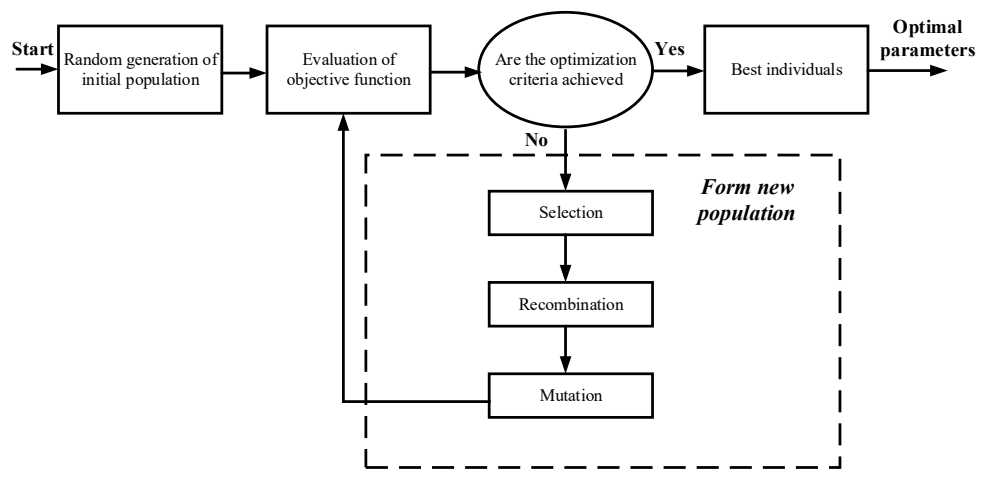

Fig.3. Block scheme of genetic algorithm. 
In the beginning, the GA randomly generates initial population of $n$ individuals (chromosomes). Then, it evaluates the objective function for initial population. In the next step, the individuals are ranked and their fitness is obtained. The algorithm for selection of individuals choses ones for reproduction, based on their relative fitness. In this manner, the most fitted individuals are used for reproduction. Selected individuals are then recombined. To form children, the parents are crossed over with cross over probability. Then mutation is applied with determinate probability. The aim of mutation is to prevent falling of all solutions in the population into a local minimum. The crossover and mutation operators are realized to yield improved children for the next generations. The objective and fitness functions for new individuals are calculated again. The new generated population is used for a further run of the algorithm. Due to random generation of initial population, to achieve global optimum it is recommended to run several times the optimization procedure. Then one can use the best solution.

The optimization problem (8) is solved by $M A T L A B^{\circledR}$ function $g a$. The 15 runs of GA procedure with initial population of 100 individuals and option 'vectorized' for fitness function are performed. The obtained PID controller parameters and the value of objective function are presented in Table1. In every run the optimization procedure stops due to that the average change in value of objective function is less than defined tolerance, which means that global or local minimum is achieved. The values of objective function for all runs are almost the same, which indicates that achieved optimum is global minimum. The values of $K_{p_{p o s}}$ and $K_{i_{p o s}}$ for all runs are close too.

Table 1. PID controller parameters.

\begin{tabular}{|c|c|c|c|c|c|c|}
\hline Run & $J(\theta)$ & $K_{p_{p a s}}$ & $K_{p_{\text {LVDT }}}$ & $K_{i_{\text {pos }}}$ & $T_{d_{\text {pos }}}$ & $T_{d_{\text {LVDT }}}$ \\
\hline 1 & $1.293778 \times 10^{6}$ & -26.8670 & -6.5600 & 0.0570 & 0.5680 & 0.0090 \\
\hline 2 & $1.2937649 \times 10^{6}$ & -26.7690 & -0.0090 & 0.0570 & 0.6000 & 0.1030 \\
\hline 3 & $1.295076 \times 10^{6}$ & -25.2080 & -0.1250 & 0.0690 & 0.8560 & 0.3760 \\
\hline 4 & $1.2939273 \times 10^{6}$ & -26.7540 & -0.0250 & 0.0590 & 0.6270 & 0.0490 \\
\hline 5 & $1.293454 \times 10^{6}$ & -25.6950 & -0.3980 & 0.0620 & 0.7930 & -0.0460 \\
\hline 6 & $1.2938889 \times 10^{6}$ & -26.5890 & 0.002 & 0.0590 & 0.6330 & 0.0330 \\
\hline 7 & $1.2940965 \times 10^{6}$ & -26.2380 & -8.8190 & 0.0610 & 0.6830 & -0.0460 \\
\hline 8 & $1.2954078 \times 10^{6}$ & -25.0060 & -12.5890 & 0.0710 & 0.8630 & 0.0800 \\
\hline 9 & $1.2955409 \times 10^{6}$ & -24.7330 & -16.2500 & 0.0720 & 0.9040 & 0.2780 \\
\hline 10 & $1.2936065 \times 10^{6}$ & -27.1200 & -0.0020 & 0.0550 & 0.5410 & 0.0140 \\
\hline 11 & $1.2936983 \times 10^{6}$ & -26.8200 & -7.7190 & 0.0560 & 0.5740 & 0.0020 \\
\hline 12 & $1.2934437 \times 10^{6}$ & -27.5520 & -0.0310 & 0.0520 & 0.4550 & 6.7350 \\
\hline 13 & $1.2942432 \times 10^{6}$ & -25.9000 & -0.5020 & 0.0620 & 0.7530 & 0.2650 \\
\hline 14 & $1.2938759 \times 10^{6}$ & -26.6070 & -7.9130 & 0.0580 & 0.6180 & 0.0050 \\
\hline 15 & $1.2958139 \times 10^{6}$ & -24.5170 & -13.7200 & 0.0730 & 0.943 & 0.2270 \\
\hline
\end{tabular}

In Fig.4 transient response of model (1) respect to cylinder piston position is presented. In Fig.5, Fig.6 and Fig.7 transient responses and control signal of control systems based on the multivariable PID controller with parameter values from Table 1 are depicted.

It is seen that the transient response of closed loop system is aperiodic and it is more 10 times faster than one for plant model. For all fifteen values of $\theta$ (vector with PID controller parameters), transient responses and control signals are very close, which again approves that the obtained minimum is global. The control signal has admissible form and does not achieve its extremal values. 


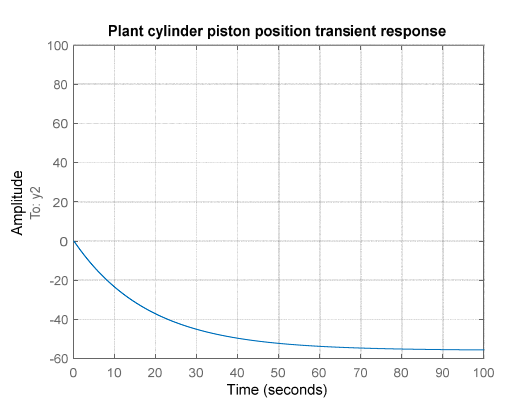

Fig.4. Plant cylinder piston position.

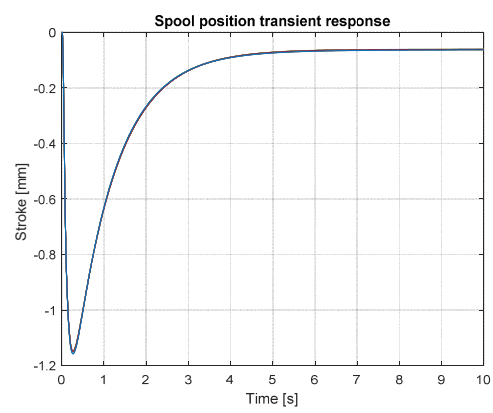

Fig.6. LVDT

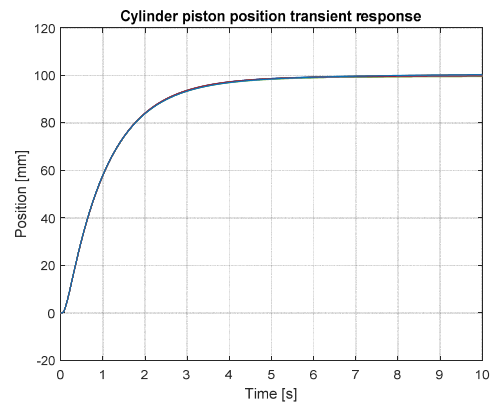

Fig.5. Control system cylinder piston position.

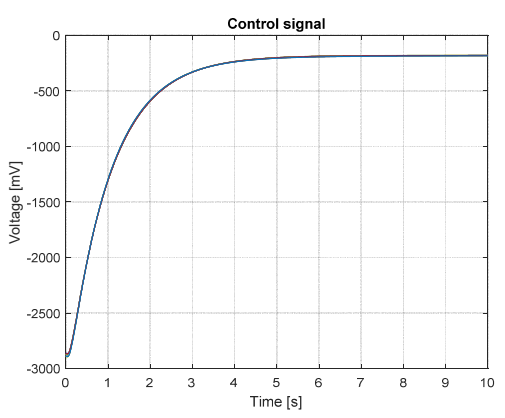

Fig.7. Control signal.

\section{Comparison of simulation and experimental results}

The tuned PID controller is embedded into the microcontroller of test rig for experimental investigation of electrohydraulic servo steering system. The implementation of controller is realized through real-time Simulink ${ }^{\circledR}$ program which is presented in Fig. 8 [10]. The main block of the model structure is a Single Precision Controller which is a subsystem for representation of the multivariable PID controller after GA-tuning. The subsystem structure is depicted in Fig.9.
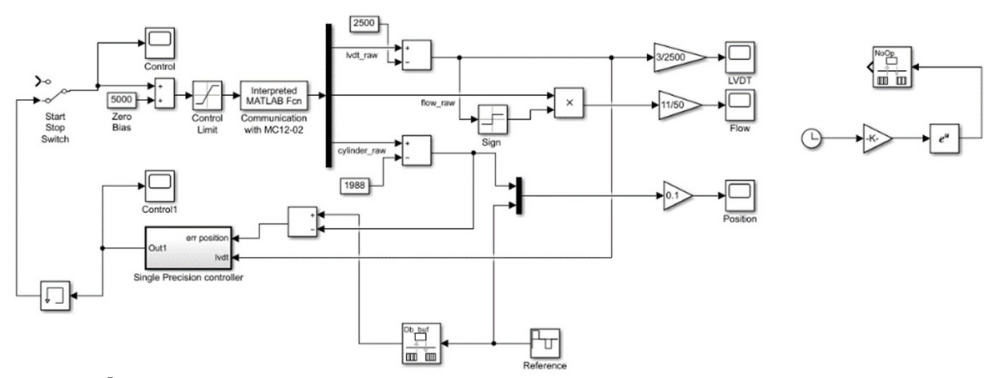

Fig.8. Simulink ${ }^{\circledR}$ model for controller implementation. 


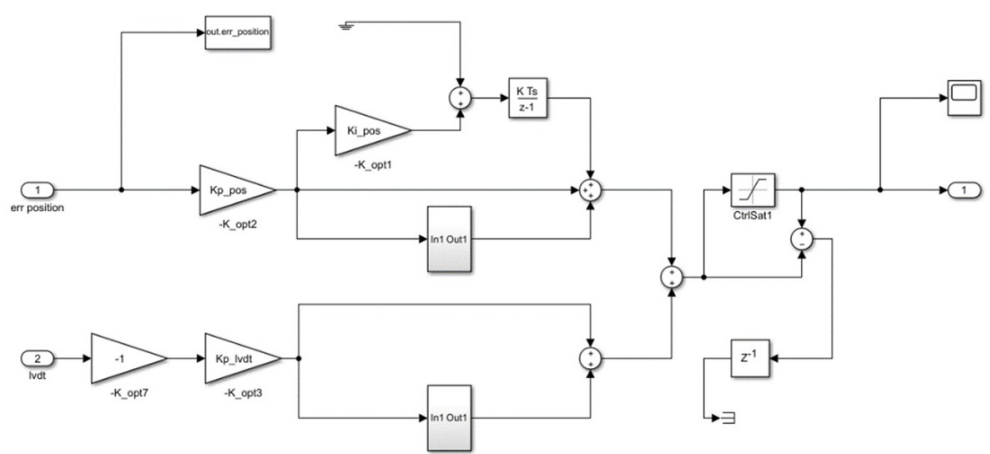

Fig.9. Subsystem of embedded multivariable PID controller.

The control performance is investigated based on several physical experiment. During the experimental tests we set a constant value $(5 \mathrm{MPa})$ of pressure load in the both chambers of the servo cylinder via counter-balance valves, which are incorporated in the hydraulic block and realized a load system.

Figure 10 compares the experimental and simulated cylinder reaction to the reference signal. The programmed reference trajectory is a rectangular signal which span the full range from -0.1 to $0.1 \mathrm{~m}$ with period of 30 seconds. For the experiments we've programmed $2^{\text {nd }}$, $3^{\text {rd }}$ and $4^{\text {th }}$ PID parameter tunings from the Table 1.

However, their performance is very similar so in the figures we show only the results from the $3^{\text {rd }}$ parameter set. Interesting effect to be observed is that experimental response is several $3 \div 4$ seconds faster than the simulated response. The experimental system with the designed PID controller demonstrates very good reference tracking with settling time around 1.5 seconds. From our other investigations on the test bench we can conclude that it is very hard to achieve such response with other control structures or heuristic manual tuning of the PID gains.

A more detailed view of the second stepwise transient is presented in Fig. 11. There is not overshot in both simulated and experimental response. The experimental response can be classified as critic aperiodic due to its small ripple above the reference, which doesn't affect the closed-loop system performance for low speed steering applications. Both simulated and experimental systems keep steady state error very small around $1 \mathrm{~mm}$. An interesting observation about the experimental system is its small sensitivity to measurement noises even though the controller reaction is very fast.

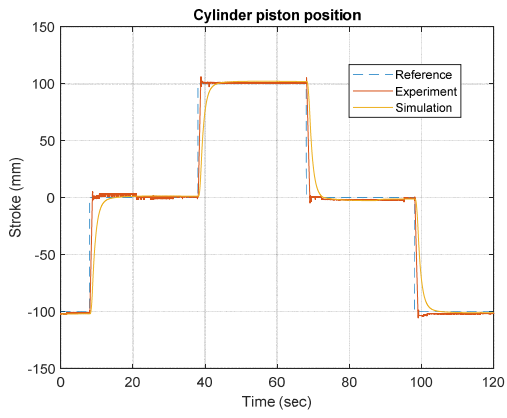

Fig.10. Transient response.

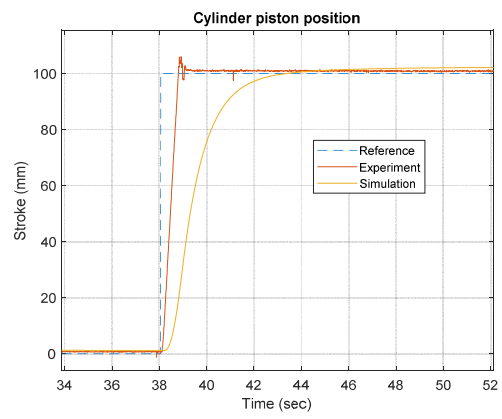

Fig.11. Detailed view of transient response. 
Comparison the control signal of the simulated and experimental closed-loop system is depicted in Fig.12. Here we can note that the effect of the measurement noise is amplified in the experimental control signal, but its amplitude is not high enough to cause oscillation in the directional spool valve. For example if the noise amplitude was high enough to cause a change in spool direction beyond the zero point, that would have been demonstrated in the cylinder response. Another observation from the control signal is that during stepwise reference change the implemented controller reacts by applying maximal voltage to the PVE block. That guarantees an optimal response in terms of rising time because of the maximal cylinder velocity. However such movement can cause overshot if not stopped at the right moment. The simulated system doesn't have such oscillations in the steady state control signal because sensor noise is not simulated.

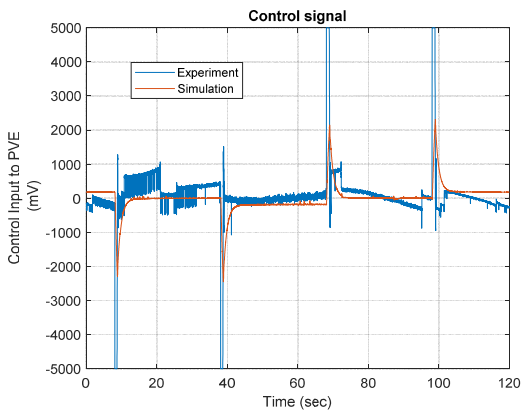

Fig.12. Plant cylinder piston position.

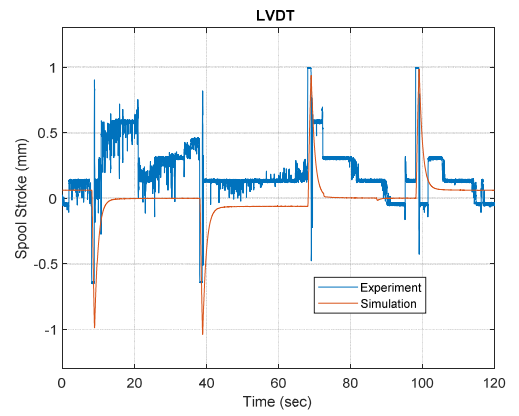

Fig.13. Control system cylinder piston position.

Comparison between the experimental and simulated response of the directional spool valve is presented in Fig.13. Its motion is sensed by the LVDT sensor which detects the change in inductance caused by the spool movement. It is important to note that spool response is very fast, allowing fast execution of the calculated control signal. That is important to achieve an accurate cylinder positioning. Also the amplitude of the simulated and experimental LVDT signal is reaching same level. There is a steady state bias in the experimental signal which is due to mechanical zero bias an unidirectional spool spring element. However, these mechanical asymmetries doesn't impact the performance of the closed-loop system, and the controller compensates for them by automatically offsetting the spool position.

\section{Conclusions}

The article presents a modern method for tuning of multivariable PID controller designed for electrohydraulic servo system intended for load-sensing power steering. The method for setting of the controller parameters is essentially an optimization procedure using a genetic algorithm to minimize the performance index. The contribution of used method is that unlike other widely used methods for tuning of PID controllers, this allows multivariable controllers to be set. The description of the algorithm including its block diagram and the results for obtained PID controller parameters during the optimization procedure are presented in detail. The tuned PID controller is embedded into the microcontroller of test rig for experimental investigation of electrohydraulic servo steering system. The performance analysis is done based on comparison between experimental and simulation results, which are presented also. 


\section{References}

1. H. Merrit, Hydraulic Control Systems, John Wiley \& Sons, Inc., ISBN 978-0-47159617-2, (1967).

2. K. Aström, T. Hagglund, PID Controllers: Theory, Design and Tuning, Second Edition, ISBN 1-55617-516-7, United States of America, (1995).

3. H. Feng, C. Yin, W. Weng, W. Ma, J. Zhou, W. Jia, Z. Zhang, Robotic excavator trajectory control using an improved GA based PID controller. Mechanical Systems and Signal Processing, 105, 153-168. doi:10.1016/j.ymssp.2017.12.014, (2018).

4. Al. Mitov, Ts. Slavov, J. Kralev, Il. Angelov, System Identification of Electro-Hydraulic Load-Sensing Servo System, 20th International Scientific Conference Engineering for Rural Development, ISSN 1691-5976, Jelgava, Latvia, (2021).

5. K. Elbayomy, J. Zongxia, Z. Huaqing, PID Controller Optimization by GA and Its Performances on the Electro-hydraulic Servo Control System, Chinese Journal of Aeronautics, 21, 4, 378-384. doi:10.1016/s1000-9361(08)60049-7, (2008).

6. T. Samakwong, W. Assawinchaichote, PID Controller Design for Electro-hydraulic Servo Valve System with Genetic Algorithm, Procedia Computer Science, 86, 91-94. doi:10.1016/j.procs.2016.05.023, (2016).

7. M. Hassan, N. Azubir, H. Nizam, S. Toha, B. Ibrahim, Optimal Design of Electric Power Assisted Steering System (EPAS) Using GA-PID Method, Procedia Engineering, 41, 614-621. doi:10.1016/j.proeng.2012.07.220, (2012).

8. Danfoss Inc., Steering, EHi steering valve, Technical Information, BC00000379enUS0202, (2018).

9. L. Ljung, System Identification: Theory for the User. Prentice-Hall, Inc., Englewood Cliffs, NJ, 2nd edition, ISBN 978-0136566953, (1999).

10. G. Todorov, K. Kamberov, T. Ivanov, Parametric optimisation of resistance temperature detector design using validated virtual prototyping approach, Case Studies in Thermal Engineering, 28, 1-9. doi:10.1016/j.csite.2021.101302 (2021).

11. Al. Mitov, Al., J. Kralev, Ts. Slavov, Il. Angelov, Model Predictive Control Design for Electro-hydraulic Power Steering Application, The 9th Mediterranean Conference on Embedded Computing - MECO'2020, ISBN 978-1-5386-5683-9, Montenegro, (2020). 